\section{Health Care-Associated Rotavirus Illness in Pediatric Inpatients in Germany, Austria, and Switzerland}

Ivo M. Foppa, DSc, MD; Wilfried Karmaus, MD, MPH; Birgit Ehlken, MSc; Martin Frühwirth, MD; Ulrich Heininger, MD; Anita Plenge-Bönig, DVM, MPH; Johannes Forster, MD, MME

A longitudinal study of health care-associated transmission of rotaviruses (RVs) in pediatric inpatients $0-48$ months old in Austria, Germany, and Switzerland showed that almost one third of all cases occurred in patients 2 months old or younger. The effectiveness of vaccination against $R V$ from 2 months of age onward remains to be evaluated.

Infect Control Hosp Epidemiol 2006; 27:633-635

Rotaviruses are an important cause of acute gastroenteritis (AGE) in young children that leads to hospitalization more often than AGE caused by other viruses.' Because rotavirus infection is highly contagious and has a short incubation period, frequent health care-associated transmission of these agents should be expected whenever effective infection control measures are not strictly followed. Although several health care-associated outbreaks have been documented, ${ }^{2,3}$ the overall frequency of such outbreaks remains unknown. Furthermore, epidemiological studies of rotavirus infection based on outbreak investigations may be subject to sampling bias. ${ }^{4}$ Previous prospective studies of health care-associated rotavirus infection in children have either monitored pediatric inpatient populations for a short period or included small patient groups. ${ }^{5,6}$ To accurately describe the epidemiological characteristics of health care-associated rotavirus infection in pediatric inpatients, we conducted a prospective longitudinal multicenter study in Germany, Austria, and Switzerland.

\section{METHODS}

The study was conducted between 1997 and 1998 in public hospitals in the 3 neighboring countries Germany, Austria, and Switzerland. In Germany, 1 study hospital each was located in Bochum, Dresden, Erlangen, and Hamburg, and 2 hospitals were located in Freiburg. One of the hospitals in Freiburg (St. Josefskrankenhaus) and the hospital in Hamburg (Wilhelmsstift) were secondary care children's hospitals, and the all other hospitals were tertiary care centers (i.e., university hospitals). In Austria, 1 hospital was a tertiary care center (Department of Pediatrics, University of Innsbruck), and the other was a secondary care hospital (Leoben). The 2 hospitals in Switzerland were university hospitals and, thus, were tertiary care centers (Geneva and Basel). Independent of this highest level of care provided, all participating hospitals had an emergency department and, thus, also provided primary care. Follow-up periods differed between study sites. In all German sites except Freiburg, periods of observation were between May 1997 and April 1998. For Freiburg, the collection of follow-up information was extended to May 1998. The sites in Austria and Germany were under observation from December 1997 to May 1998.

Children up to 48 months of age who were admitted to any of the 10 study hospitals during the study period for reasons unrelated to rotavirus infection, who developed AGE, and whose parents gave written consent for their children to participate were included in the study. ${ }^{1}$ The protocol had been approved by all relevant institutional review boards and ethics committees. All study hospitals followed guidelines of the German Society for Pediatric Infectious Diseases, which are based on the current guidelines on contact precautions from the Red Book. ${ }^{7}$ Stool samples from all children who developed diarrhea were first examined by an antigen-detection enzymelinked immunosorbent assay (TestPAck; Abbott), in accordance with the manufacturer's instructions. A case of health care-associated rotavirus infection was defined by a positive result of the stool-antigen test for a child whose symptoms started no sooner than 48 hours after admission. Information about patient's age group $(0-12,13-24,25-36$, or $37-48$ months) and study site-specific numbers of hospital-days were obtained from hospitalization records. Information on the number of children at risk for rotavirus infection at any given time (number of patient-days of observation) was collected retrospectively from patient records. Only cases of rotavirus infection that occurred during months for which such information was available were included in the study. We calculated pointwise exact $95 \%$ Poisson confidence intervals (CIs) for incidence rates. ${ }^{8}$ Proportions were compared using the $\chi^{2}$ test for $2 \times 2$ tables where expected cell values were greater than 5 and Fisher's exact test otherwise. ${ }^{9}$ Means were compared using Student's 2-sample $t$ test. For proportions, asymptotic binomial $95 \% \mathrm{CIs}$ were calculated. Statistical analyses were performed using SAS (version 9.1; SAS Institute) and Mathematica (version 4.1; Wolfram Research).

\section{RESULTS}

Of 510 stool samples collected from the 510 children with AGE, 266 (52.2\%) tested positive for rotavirus. The mean age of children with a positive test result was similar to that of children with a negative stool test result (7.4 vs. 6.7 months; $P=.39$ ). Among the children with a positive stool test result, one fifth ( $n=54$ ) were 30 days old or younger at diagnosis, almost one third ( $n=78$ ) were 2 months old or younger, and the majority ( $n=218$ ) were less than 1 year old. For 2 children, no information on age was available. Of all rota- 
TA BLE. Age-Specific Incidences of Acute Gastroenteritis Due to Rotavirus (RV) and Not Due to RV in Patients 0-48 Months Old at the 9 Study Sites

\begin{tabular}{|c|c|c|c|c|c|c|c|c|c|c|c|c|c|c|c|}
\hline \multirow[b]{2}{*}{ Study site } & \multicolumn{3}{|c|}{ All ages } & \multicolumn{3}{|c|}{$0-12$ mo of age } & \multicolumn{3}{|c|}{$13-24$ mo of age } & \multicolumn{3}{|c|}{$25-36 \mathrm{mo}$ of age } & \multicolumn{3}{|c|}{$37-48$ mo of age } \\
\hline & Non-RV & RV & $\mathrm{PD}$ & Non-RV & RV & $\mathrm{PD}$ & Non-RV & RV & $\mathrm{PD}$ & Non-RV & RV & PD & Non-RV & RV & $\mathrm{PD}$ \\
\hline Freiburg & 25 & 77 & 23,389 & 15 & 65 & 15,934 & 8 & 8 & 3,279 & 2 & 3 & 2,208 & 0 & 1 & 1,968 \\
\hline Dresden & 32 & 7 & 15,748 & 30 & 6 & 2 & 2 & 0 & 2,079 & 0 & 1 & 1,092 & 0 & 0 & 771 \\
\hline Bochum & 28 & 28 & 15,251 & 26 & 28 & 11,347 & 1 & 0 & 2,144 & 1 & 0 & 886 & 0 & 0 & 874 \\
\hline Hamburg & 44 & 47 & 29,016 & 27 & 34 & 17,294 & 8 & 9 & 5,635 & 7 & 3 & 3,328 & 2 & 1 & 2,759 \\
\hline Innsbruck & 10 & 9 & 8,675 & 10 & 8 & 5,947 & 0 & 1 & 1,254 & 0 & 0 & 901 & 0 & 0 & 574 \\
\hline Basel & 11 & 8 & 8,019 & 10 & 8 & 6,001 & 1 & 0 & 951 & 0 & 0 & 761 & 0 & 0 & 305 \\
\hline Geneva & 7 & 9 & 6,750 & 4 & 6 & 5,477 & 3 & 3 & 646 & 0 & 0 & 369 & 0 & 0 & 259 \\
\hline Total & 187 & 215 & 138,893 & 145 & 174 & 94,332 & 27 & 26 & 20,806 & 13 & 10 & 13,017 & 2 & 5 & 10,738 \\
\hline
\end{tabular}

NOTE. Data are no. of cases or no. of patient-days of observation (PDs).

virus-positive cases of $\mathrm{AGE}$, almost one half $(47.7 \%)$ were diagnosed within 5 days after admission. Denominator information was available for 402 cases of AGE accounting for 138,893 hospital-days, including cases in 215 patients with rotavirus-positive stool tests (Table). This corresponds to an overall incidence rate of 1.5 cases/1,000 hospital-days (95\% $\mathrm{CI}, 1.3-1.8)$. However, incidence rates of rotavirus infection varied widely among study locations. The highest and lowest observed average incidence rates differed by a factor of almost 20. To examine epidemiologic patterns of rotavirus infection, we analyzed location-specific incidence rates by month (Figure). In some study sites, we observed health care-associated rotavirus transmission during a major part of the study pe- riod, and transmission was relatively intense (in Freiburg, Bochum, and Leoben). At other sites, we detected health careassociated rotavirus transmission only during a minor part of the study period, and transmission remained relatively low (Erlangen and Dresden). The remaining sites exhibited an intermediate level of transmission. The highest local monthly incidence rate that we observed in our study was 10.8 cases/ 1,000 hospital-days (95\% CI, 6.2-17.5) (Freiburg, April 1998). This rate translates into a $7 \%$ risk of acquiring rotavirus infection during a 1-week hospital stay. The corresponding rate was even higher in infants ( 15.4 cases/1,000 hospitaldays [95\% CI, 8.4-25.8]), resulting in a more than $10 \%$ risk during a 1-week hospital stay.


FIGURE. Monthly incidence rates ( $Y$-axes, no. of cases per 1,000 hospital-days at risk) in the 9 study locations. The exact upper $95 \%$ Poisson confidence interval is shown as an error bar. The letters along the $X$-axes represent months, starting with May 1997 and ending with May 1998. The cutoff upper error bar (Freiburg, May 1998) represents a value of 21.5. 


\section{I SCUSSION}

In this prospective multicenter study, the average observed incidence rate of rotavirus-positive AGE was 1.5 cases $/ 1,000$ children-days, accounting for more than half of all health care-associated cases of AGE. Although we found evidence of health care-associated rotavirus transmission in all study sites, incidence rates varied widely both between centers and over time.

Although a number of investigations have described outbreaks of health care-associated rotavirus infection in pediatric settings, ${ }^{10-12}$ such studies did not provide information on the overall incidence of health care-associated rotavirus infections. Several studies have determined the risk of health care-associated rotavirus infection in children, ${ }^{5,6,13-16}$ but only the study by Pacini et al. ${ }^{5}$ estimated incidence density during a 4.5 -month period. Those researchers detected 1.1 cases of health care-associated AGE per 1,000 children-days, of which $44 \%$ were due to rotavirus, corresponding to an estimated incidence rate of less than 0.5 cases of rotavirus infection per 1,000 children-days. This is somewhat less than the average incidence rate that we observed but corresponds well with some local incidence rates observed in our study. Among all cases of AGE acquired in the hospital, more than half were caused by rotavirus. This is a larger proportion than has been reported previously. ${ }^{17}$ Only longitudinal epidemiological studies of rotavirus infection similar to our study will help to attribute differences in rotavirus incidence between study sites to meaningful epidemiologic differences, to epidemic variability, or both.

Given the short incubation period of rotavirus infection and the fact that we excluded patients who developed symptoms within 48 hours of admission, rotavirus illness in the vast majority of our cases can be assumed to have been hospital acquired. Therefore, we do not believe that the incidence density estimates reported here are significantly inflated by the inclusion of community-acquired cases. The estimated risk of health care-associated rotavirus infection during a 1week hospital stay of $10 \%$ appears to be substantial. In combination with the high burden on healthcare systems imposed by rotavirus infection, ${ }^{17}$ this finding underscores the importance of preventive measures, including hospital hygiene and infection control. However, because almost one third of the children identified in the present study were 2 months old or younger, the effect on transmission dynamics of rotaviruses of immunization starting at the age of 2 months remains to be evaluated.

\section{ACKNOWLEDGMENT}

Collection of the data for the study was funded by Wyeth-Pharma GmbH, Münster, Germany.

From the Department of Epidemiology and Biostatistics, Arnold School of Public Health, Columbia, South Carolina (I.M.F. and W.K.), Medical
Economics Research Group, Munich (B.E.), the Department of Clinical Microbiology and Hygiene, Institut für Hygiene und Umwelt, Hamburg (U.H.), and the Department of Pediatrics, St. Josefskrankenhaus, Freiburg (J.F.), Germany; the Department of Pediatrics, Medical University Innsbruck, Innsbruck, Austria (M.F.); and University Children's Hospital, Basel, Switzerland (A.P.-B.).

Address reprint requests to Ivo M. Foppa, Department of Epidemiology and Biostatistics, Arnold School of Public Health, 800 Sumter St., Columbia, SC 29208 (ifoppa@sc.edu).

Received November 16, 2005; accepted January 27, 2006; electronically published May 31, 2006.

(C) 2006 by The Society for Healthcare Epidemiology of America. All rights reserved. 0899-823X/2006/2706-0018\$15.00.

\section{REFERENCES}

1. Fhlken B, Laubereau B, Karmaus W, Petersen G, Rohwedder A, Forster J. Prospective population-based study on rotavirus disease in Germany. Acta Paediatr 2002; 91:769-75.

2. Widdowson MA, van Doornum GJ, van der Poel WH, et al. An outbreak of diarrhea in a neonatal medium care unit caused by a novel strain of rotavirus: investigation using both epidemiologic and microbiological methods. Infect Control Hosp Epidemiol 2002; 23:665-70.

3. Ratner AJ, Neu N, Jakob $\mathrm{K}$, et al. Healthcare associated rotavirus in a pediatric hospital. Infect Control Hosp Epidemiol 2001; 22:299-301.

4. Berner R, Schumacher RF, Hameister S, Forster J. Occurrence and impact of community-acquired and healthcare associated rotavirus infectionsa hospital-based study over 10 y. Acta Paediatr Suppl 1999; 88:48-52.

5. Pacini DL, Brady MT, Budde CT, Connell MJ, Hamparian VV, Hughes $\mathrm{JH}$. Healthcare associated rotaviral diarrhea: pattern of spread on wards in a children's hospital. J Med Virol 1987; 23:359-66.

6. Ringenbergs ML, Davidson GP, Spence J, Morris S. Prospective study of healthcare associated rotavirus infection in a paediatric hospital. Aust Paediatr J 1989; 25:156-60.

7. Pickering LK, ed. Red Book: 2003 Report of the Committee of Infectious Diseases, 26th ed. Elk Grove, IL: American Academy of Pediatrics, 2003: $534-6$.

8. Ulm K. A simple method to calculate the confidence interval of a standardized mortality ratio (SMR). Am J Epidemiol 1990; 131:373-5.

9. Agresti A. Categorical Data Analysis. New York: John Wiley \& Sons, 1990.

10. Glasgow JF, McClure BG, Connolly JH, O'Neill HJ. Healthcare associated rotavirus gastroenteritis in a neonatal nursery. Ulster Med J 1978; 47:50-6.

11. Gerna G, Forster J, Parea M, et al. Healthcare associated outbreak of neonatal gastroenteritis caused by a new serotype 4, subtype $4 \mathrm{~B}$ human rotavirus. J Med Virol 1990; 31:175-82.

12. Widdowson MA, van Doornum GJ, van der Poel WH, de Boer AS, Mahdi $U$, Koopmans M. Emerging group-A rotavirus and a healthcare associated outbreak of diarrhoea. Lancet 2000; 356:1161-2.

13. Ford-Jones EL, Mindorff CM, Gold R, Petric M. The incidence of viralassociated diarrhea after admission to a pediatric hospital. Am J Epidemiol $1990 ; 131: 711-8$.

14. Vial PA, Kotloff KL, Losonsky GA. Molecular epidemiology of rotavirus infection in a room for convalescing newborns. $J$ Infect Dis 1988 ; 157 : 668-73.

15. Harrington $\mathrm{M}$, Butler $\mathrm{K}$, Cafferkey $\mathrm{M}$. Rotavirus infection in hospitalised children: incidence and impact on healthcare resources. Ir J Med Sci 2003; 172:33-6

16. Mastretta E, Longo P, Laccisaglia A, Balbo L, Russo R, Mazzaccara A, Gianino P. Effect of Lactobacillus $\mathrm{GG}$ and breast-feeding in the prevention of rotavirus healthcare associated infection. J Pediatr Gastroenterol Nutr 2002; 35:527-31.

17. Fischer TK, Bresee JS, Glass RI. Rotavirus vaccines and the prevention of hospital-acquired diarrhea in children. Vaccine 2004; 22(Suppl 1):S49-S54. 